\title{
Automotive radar - investigation of mutual interference mechanisms
}

\author{
M. Goppelt ${ }^{1}$, H.-L. Blöcher ${ }^{2}$, and W. Menzel $^{1}$ \\ ${ }^{1}$ Institute of Micowave Techniques, University of Ulm, 89081 Ulm, Germany \\ ${ }^{2}$ Group Research \& Advanced Engineering, Daimler AG, 89081 Ulm, Germany
}

\begin{abstract}
In the past mutual interference between automotive radar sensors has not been regarded as a major problem. With an increasing number of such systems, however, this topic is receiving more and more attention. The investigation of mutual interference and countermeasures is therefore one topic of the joint project "Radar on Chip for Cars" (RoCC) funded by the German Federal Ministry of Education and Research (BMBF). RoCC's goal is to pave the way for the development of high-performance, low-cost $79 \mathrm{GHz}$ radar sensors based on Silicon-Germanium (SiGe) Monolithic Microwave Integrated Circuits (MMICs).

This paper will present some generic interference scenarios and report on the current status of the analysis of interference mechanisms.
\end{abstract}

\section{Introduction}

In the automotive field, radar sensors are key components for comfort and safety functions, for example adaptive cruise control (ACC) or collision mitigation systems (CMS). With an increasing number of automotive radar sensors operated close to each other at the same time, radar sensors may receive signals from other radar sensors. The reception of foreign signals (interference) can lead to problems such as ghost targets or a reduced signal-to-noise ratio. Figure 1 shows such a simple automotive interference scenario with direct interference from an oncoming vehicle.

Up to now, interference has not been considered as a major problem because the percentage of vehicles equipped with radar sensors and therefore the probability of interference was low, and the sensors were used mainly for comfort functions. In this case it may be sufficient to detect interference and turn off the function for the duration of the interference. On the contrary, safety functions of future systems require very low failure rates. So in spite of a predicted higher number of radar systems, the probability of interference-induced

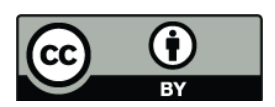

Correspondence to: M. Goppelt

(markus.goppelt@uni-ulm.de) problems has to be reduced considerably. Therefore effective countermeasures have to be introduced to minimize mutual interference even with high traffic density (e.g. in large cities) and a rising percentage of vehicles equipped with radar sensors.

In Germany currently three frequency bands are assigned to automotive radar applications:

- $24 \mathrm{GHz}$ ultra-wideband (UWB)

- 76-77 GHz

- $77-81 \mathrm{GHz}$

In addition, some systems also are operated in the $24 \mathrm{GHz}$ ISM band. Detailed information about the frequency bands can be found in Table 1 .

New vehicles can be equipped with $24 \mathrm{GHz}$ UWB sensors only until 2013. From then on, the $79 \mathrm{GHz}$ frequency band with up to $4 \mathrm{GHz}$ bandwidth will replace the $24 \mathrm{GHz}$ band.

From 2004 to 2007, the Daimler AG took part in the German Federal Ministry of Education and Research (BMBF) funded joint project KOKON. Aim of the project was to demonstrate the feasibility of $79 \mathrm{GHz}$ automotive radar sensors with SiGe-based MMICs. Presently, the Daimler AG contributes to the successor project "Radar on Chip for Cars" (RoCC) running from 2008-2011.

Special emphasis of this projects lies on the following items:

- reducing cost and size

- improving the radio-frequency (RF) packaging technology

- improving sensor performance

- improving sensor reliability

The last item includes the investigation of mutual interference and interference minimization techniques (countermeasures). The specific tasks are 
Table 1. Frequency bands and equivalent isotropically radiated power (EIRP) for automotive radar applications in Germany.

\begin{tabular}{lcccc}
\hline & $\begin{array}{c}24 \mathrm{GHz} \\
\text { UWB } \\
\text { short-range }\end{array}$ & $\begin{array}{c}24 \mathrm{GHz} \\
\text { ISM } \\
\text { short-range }\end{array}$ & long-range & short-range \\
\hline frequency range (GHz) & $21.650-26.650$ & $24.000-24.250$ & $76.000-77.000$ & $77.000-81.000$ \\
\hline $\begin{array}{l}\text { center frequency (GHz) } \\
\text { max. average power (EIRP) } \\
(\mathrm{dBm} / \mathrm{MHz})\end{array}$ & 24.150 & 24.125 & 76.500 & 79.000 \\
\hline $\begin{array}{l}\text { max. average power (EIRP) } \\
(\mathrm{dBm})\end{array}$ & -41.3 & & -9 \\
\hline $\begin{array}{l}\text { max. peak power (EIRP) } \\
(\mathrm{dBm})\end{array}$ & & & 50 & \\
\hline
\end{tabular}

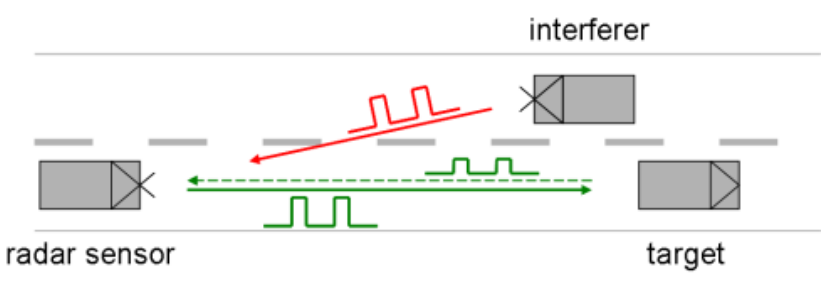

Fig. 1. Simple automotive interference scenario with one target and one interferer.

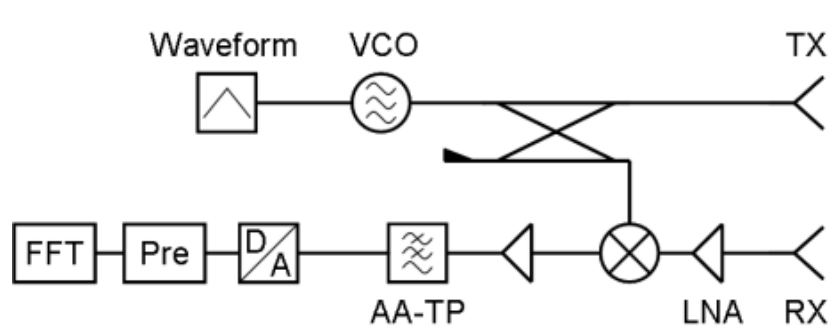

Fig. 2. Block diagram of a simple generic FMCW radar.

- to identify interference mechanisms,

- to find efficient countermeasures and

- to test countermeasures in the laboratory and with radar sensors in vehicles.

At the beginning of Sect. 2 of this paper, two radar architectures are defined, and the analysis approach to interference mechanisms between different types of radar sensors is outlined. Sections 2.1 to 2.4 illustrate the interference mechanisms. Target and interference power levels are analyzed in Sect. 2.5 for the simple interference scenario in Fig. 1.

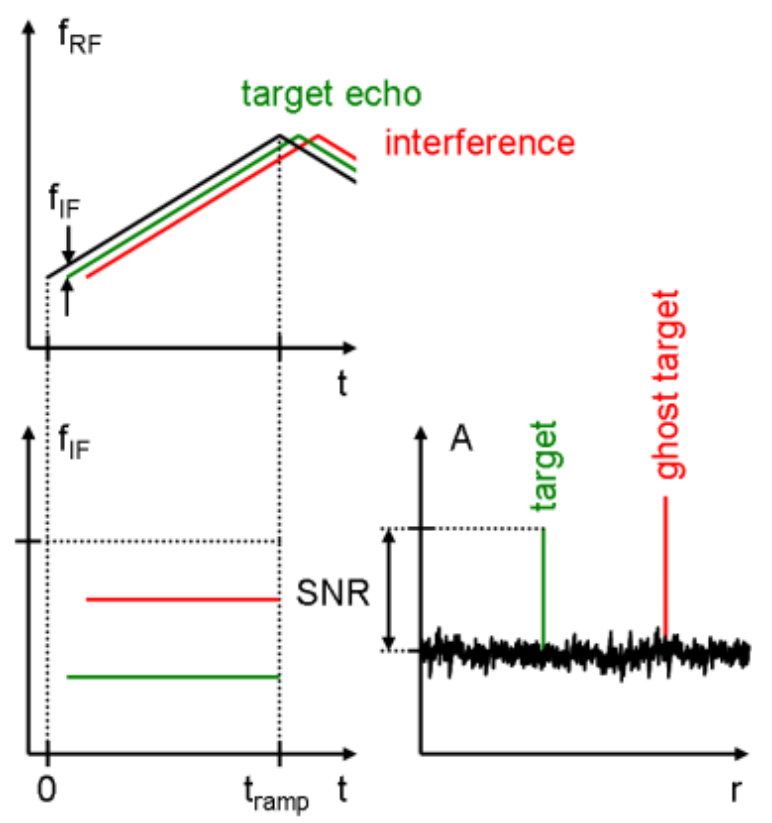

Fig. 3. Diagrams illustrating a case where an FMCW radar interferes with another FMCW radar generating a ghost target. The upper diagram shows the frequency course of the local oscillator signal (black) and the signals received by the receive (RX) antenna. The lower left-hand diagram shows the down-converted IF frequencies as a function of time, the lower right-hand diagram the Fourier transform of the IF signal.

\section{Interference mechanisms}

Since there are many different radar sensors on the automotive market, it was decided to start the analysis of interference mechanisms with a simple generic frequencymodulated continuous wave (FMCW) radar (Fig. 2) and a simple generic pulsed radar with correlation receiver (Fig. 10). 


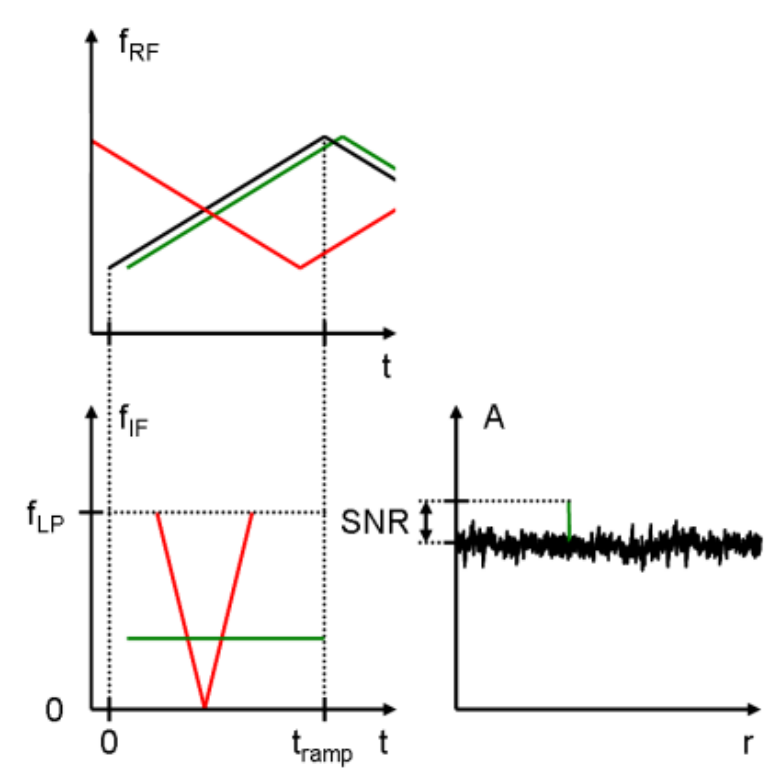

Fig. 4. Diagrams illustrating a case with interference between two FMCW radars causing a uniform increase of the noise floor.

In a first step the interference mechanisms between the different types of radar were roughly identified, followed by simulations and measurements to verify the approximations and to develop a more precise understanding of the interference mechanisms. The last item, however, has not yet been completed. Previous research on interference mechanisms had been conducted by

- Brooker (2007): Investigation of the interference between an FMCW radar and a pulsed radar and propositions for interference minimization techniques.

- Oprisan and Rohling (2005): Analysis of interference effects between continuous wave (CW), frequency shift keying (FSK), frequency-modulated continuous wave (FMCW), frequency-modulated shift keying (FMSK) and pulsed radars. Some interference reduction techniques are proposed.

- Tullsson (1997): Investigation of the interference sensitivity of an automotive FMCW radar and analysis of interference elimination techniques.

\subsection{FMCW radar with FMCW interference}

To illustrate the effects of interference from an FMCW radar on an FMCW radar, two cases were selected. Both radars have the same triangular FMCW modulation (same ramp duration and sweep bandwidth). In the first case (Fig. 3) the interference leads to a constant intermediate frequency (IF) generating a second line in the radar range profile. The second line then is interpreted as an additional target (ghost target).

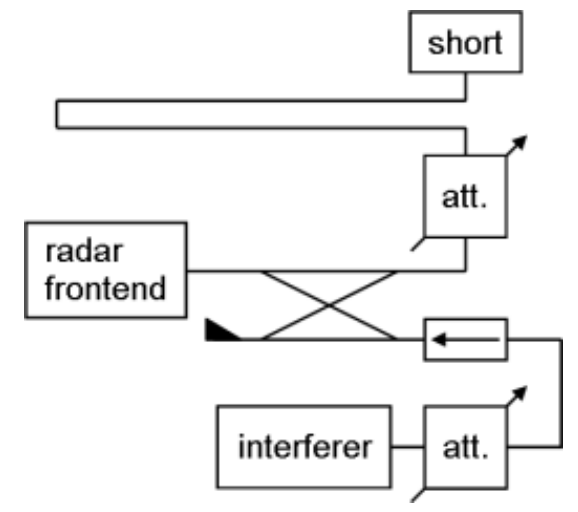

Fig. 5. Block diagram of the waveguide-based measurement setup to demonstrate the effects of interference on an FMCW radar.

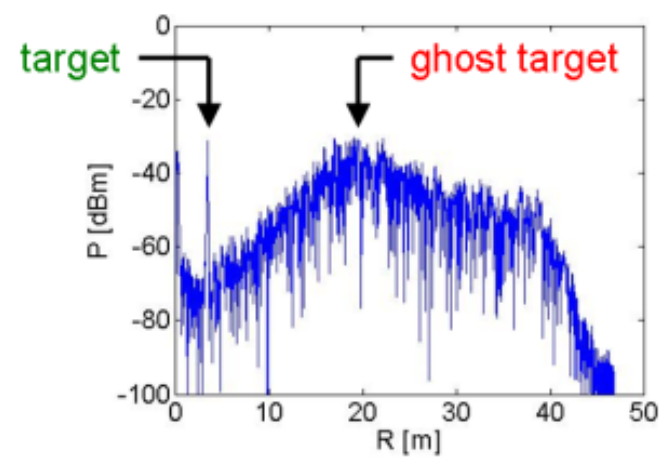

Fig. 6. Measured FMCW radar range profile with a ghost target generated by interference from another FMCW radar.

In the second case (Fig. 4) the frequency ramp of the interfering signal starts earlier in time, consequently the crossing of the ramps lead to an IF signal with fast V-shaped frequency variation over time, resulting in a uniform increase of the noise floor over the whole IF-band. Thus, the target signal-to-noise-ratio can be reduced considerably.

Both cases were demonstrated in the laboratory using a waveguide-based measurement setup (Fig. 5). The target was simulated using an approximately three meters long waveguide terminated with a short circuit. The interfering signal was added to the reflected signal using a directional coupler. Figure 6 shows the result for the first case (parallel frequency ramps). The ghost target's broad spectrum originates from the fact that there is no correlation between the phase noise of the interfering signal and of the radar's local oscillator signal.

For the second case, a CW signal was coupled into the receiver of the FMCW radar. In the frequency domain, the $\mathrm{CW}$ signal intersects the ramps of the FMCW radar just like the interfering FMCW signal in Fig. 4. The upper diagram of Fig. 7 shows the resulting IF signal in the time domain. The IF signal generated by the interfering $\mathrm{CW}$ signal is short in relation to the ramp duration. This is due to the fact that the 

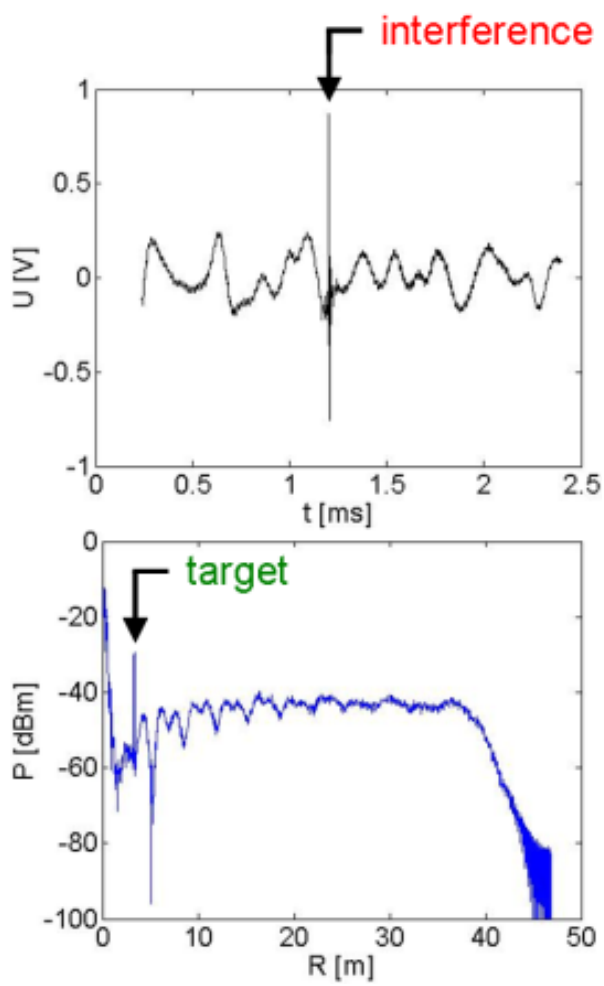

Fig. 7. Measured FMCW radar IF signal and range profile with $\mathrm{CW}$ interference.

radar's IF bandwidth is much smaller than the radar's frequency deviation (e.g. by a factor of 1000). The IF noise floor of the radar without interference is below $-60 \mathrm{dBm}$, the noise floor increases to about $-45 \mathrm{dBm}$ due to the interfering $\mathrm{CW}$ signal as can be seen in the lower diagram of Fig. 7.

\subsection{FMCW radar with pulsed interference}

A similar situation results, when a pulse modulated signal interferes with an FMCW radar. The spectrum of a pulsed signal consists of equidistant lines with a $\sin (x) / x$ envelope for rectangular pulses. This is depicted in the left part of Fig. 8. Some lines intersect the FMCW radar ramp leading to a short IF signal with V-shaped frequency course similar to the case shown in Fig. 4.

An exemplary measurement with pulsed signal interference is shown in Fig. 9. It can be seen that the resulting IF signal (upper diagram) is wider than in the $\mathrm{CW}$ case (upper diagram of Fig. 7). The resulting increase in the noise floor is not as uniform as in the $\mathrm{CW}$ case (lower diagrams of Figs. 7 and 9).

\subsection{Pulsed radar with FMCW interference}

Pulsed radars with correlation receiver, as typically used for automotive applications and also selected for this investigation, work similar to sampling oscilloscopes. The time delay
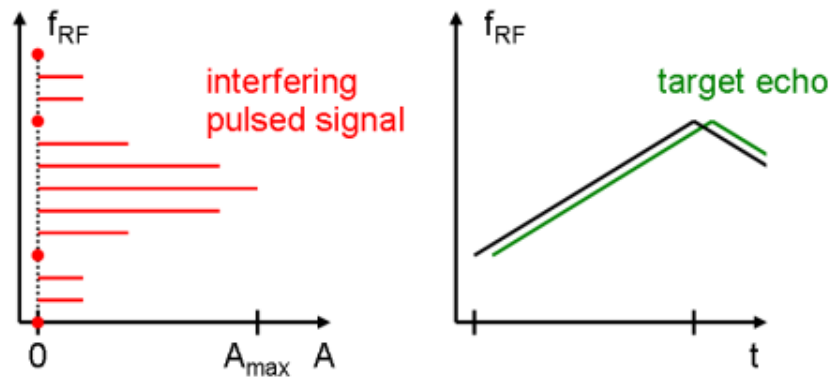

Fig. 8. Diagrams illustrating the case of pulsed signals interfering with an FMCW radar. The left-hand diagram shows the spectrum of a pulsed signal, the right-hand diagram shows the FMCW radar signals. Some of the equidistant spectral lines of the interfering pulsed signal intersect the FMCW radar ramp.
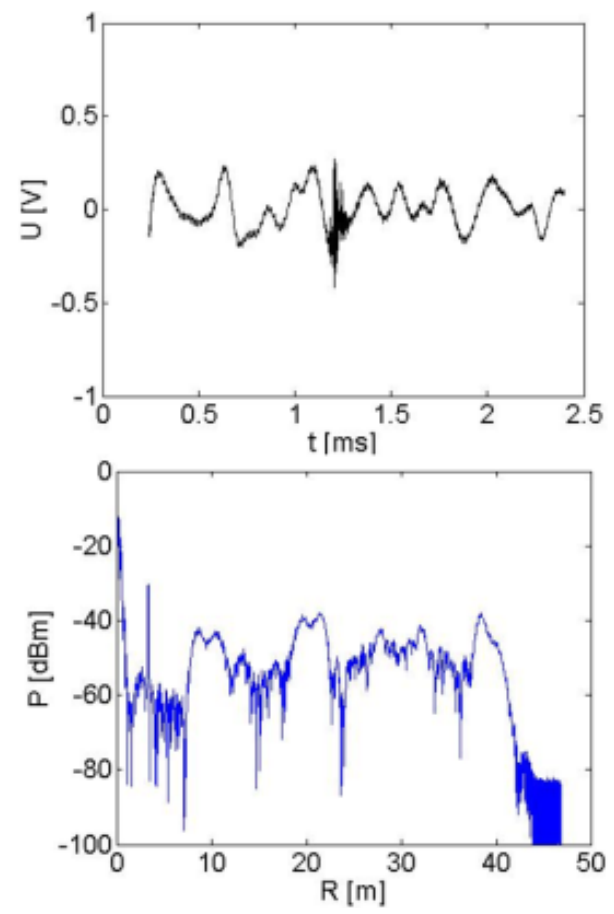

Fig. 9. Measured FMCW radar IF signal and range profile with pulsed signal interference.

$t_{\mathrm{D}}$ (Fig. 10) is successively increased until the maximum signal flight time of interest is reached. An IF signal (in principal a correlation function) appears at the mixer output if a radar signal delay and $t_{\mathrm{D}}$ are equal. This is done in such a way that every range cell is sampled several times, and the resulting output signals are integrated in a lowpass filter where the resulting amplitude is detected. Following this, the sampling process starts again with the lowest time delay. In practice, the mixer in Fig. 10 has to be substituted by a pair of mixers to get the quadrature signals. A pulsed radar sensor with correlation receiver is described in detail in a publication by Gresham et al. (2004). 


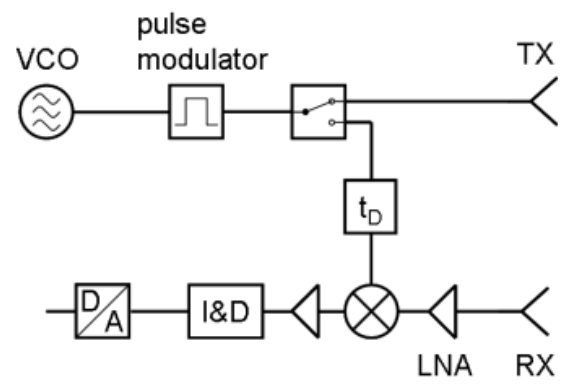

Fig. 10. Block diagram of a simple generic (coherent) pulsed radar with correlation receiver.
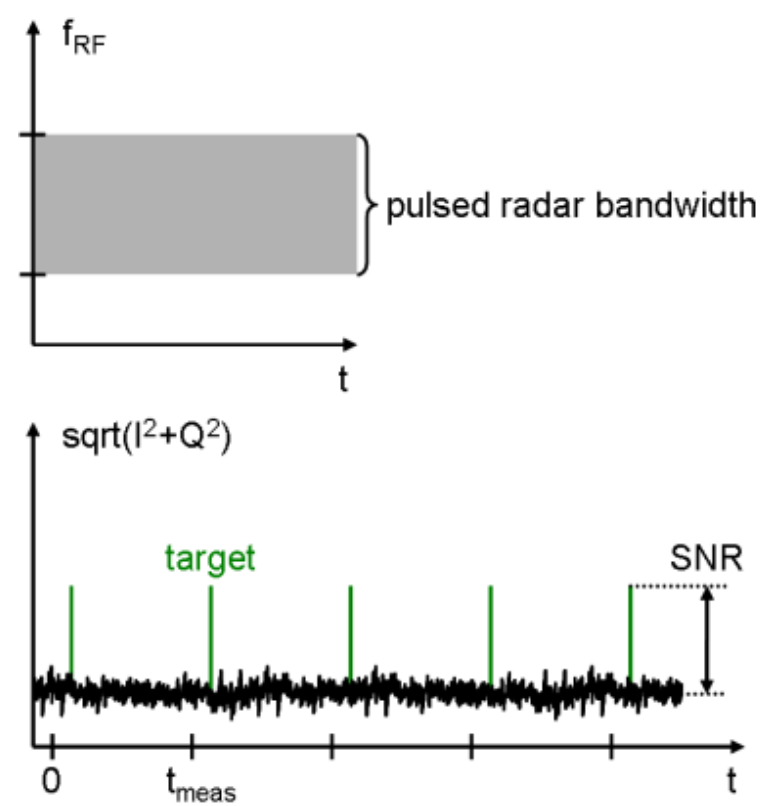

Fig. 11. Reception bandwidth and range profile of the pulsed radar without interference.

Figure 11 shows the receiver bandwidth and the range profile of the pulsed radar without interference. The amplitudes in the lower diagram are the absolute values of the complex I-Q output of the mixer.

The effect of an interference from a narrowband FMCW radar on this type of radar is a uniform increase in the noise floor (lower diagram of Fig. 12). The target signal-to-noise ratio decreases accordingly.

\subsection{Pulsed radar with pulsed interference}

Interference from an identical pulsed radar with the same pulse repetition frequency (PRF) generates a ghost target at a constant "distance" (Fig. 13).

A slightly different PRF results in a moving ghost target since the time difference between the transmitted pulse and the interfering pulse increases or decreases from pulse repetition period to pulse repetition period. Figure 14 displays this
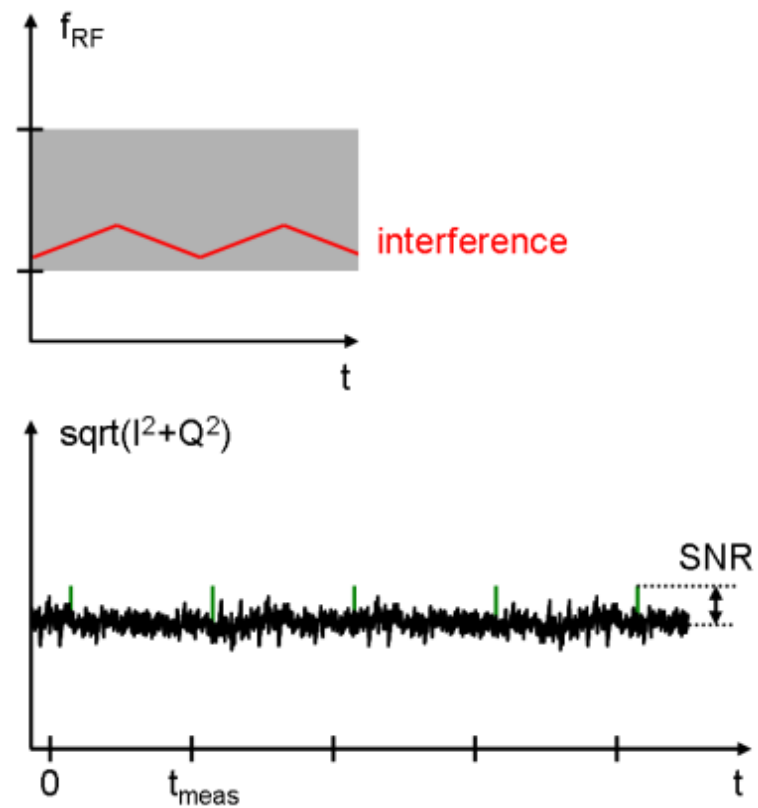

Fig. 12. Reception bandwidth and range profile of a pulsed radar with interference by a narrowband FMCW signal causing a uniform increase of the noise floor and therefore a reduction of the target signal-to-noise-ratio (SNR).

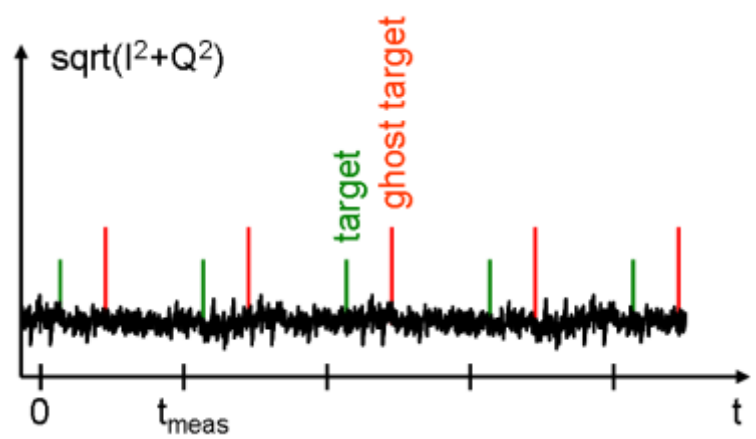

Fig. 13. Range profile of a pulsed radar with interference from an identical pulsed radar with the same PRF generating a stationary ghost target.

situation for a slightly lower PRF resulting in a ghost target with increasing distance. This diagram, however, does not yet take into account the effect of integration in the correlation receiver. Depending on the actual difference in PRF, less interference pulses may be integrated, leading to a possibly improved situation.

\subsection{Consideration of the interference power level}

So far, the power levels of the target echo and the interfering signal have not been regarded in detail. To start with an analysis of power levels, the simple interference scenario in Fig. 1 has been considered. Simplified radiation diagrams with constant antenna gain in a given sector were used as 


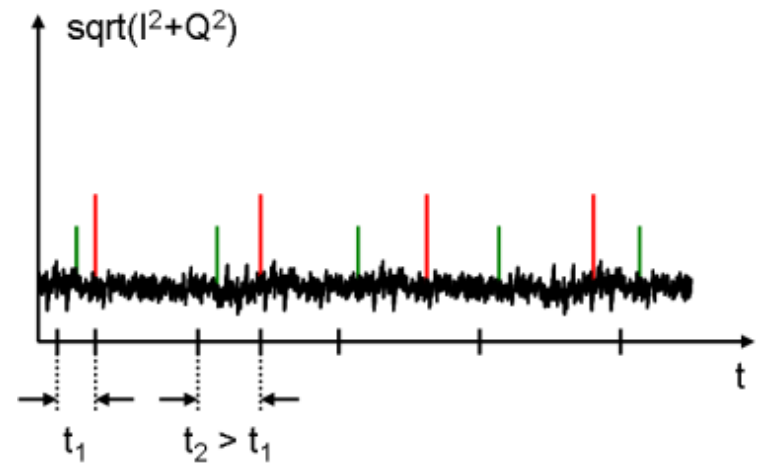

Fig. 14. Range profile of a pulsed radar with interference from an identical pulsed radar with slightly lower PRF generating a ghost target with increasing distance.

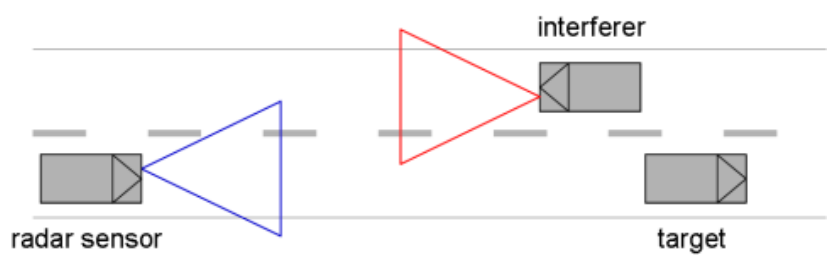

Fig. 15. Simple automotive interference scenario with simplified radiation diagrams. The interferer radiates directly into the radar sensor's receiver.

depicted in Fig. 15. The positions of the radar sensors on the vehicles as well as widths and orientations of the antenna beams are just one example. Power levels then are calculated using Friis' formula.

Figure 16 shows the simulated power levels at the antenna port of the receiving radar. The interference lasts as long as the radar sensors "see" each other. The target level is constant since it is assumed that the target vehicle is at a constant distance. As the interfering radar radiates directly into the receiver, the interference level can be much higher than the target level (more than $60 \mathrm{~dB}$ above the target level). This high interference level may have different effects. On the one hand, it may drive receiver components into saturation or into their nonlinear regime, resulting in unwanted harmonics. On the other hand, the interference signals will be further processed and can appear as ghost targets or as an increased noise level, reducing the desired SNR of the receiving radar. Some further investigations are necessary, however, including the respective analogue and digital signal processing; this may reduce the influence of the interference to some extent.

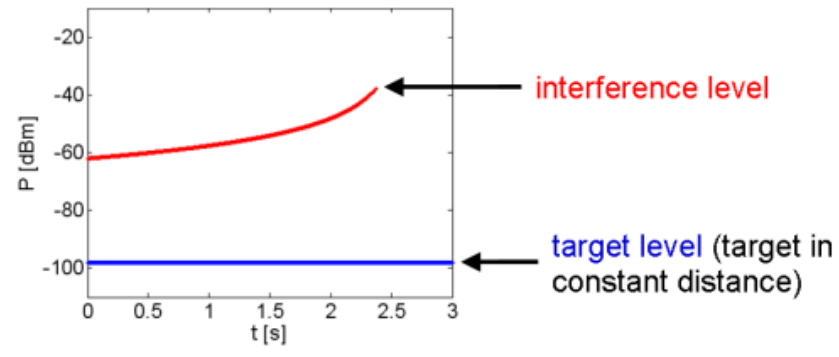

Fig. 16. Target and interference power level at the radar sensor's receive $(\mathrm{RX})$ antenna port for the scenario in Fig. 15.

are used increasingly for safety functions. On the other hand, traffic density and percentage of vehicles equipped with radar sensors will increase considerably. Interference mechanisms have to be precisely understood in order to design and to verify the effectiveness of countermeasures to minimize mutual interference.

This paper has described some fundamental interference effects appearing in a scenario with FMCW and pulsed radar sensors, partly by a simple analysis using generic radar sensors, partly by first experiments using radar frontends built by the University of Ulm.

The two major effects detected so far are the appearance of ghost targets and an increase of the noise or interference level in the radar receiver. Concerning the interference levels, very high signal strengths can occur if two sensors directly face each other.

Acknowledgements. The authors wish to thank the German Federal Ministry of Education and Research (BMBF) for funding this work within the RoCC project.

\section{References}

Brooker, G.: Mutual Interference of Millimeter-Wave Radar Systems, IEEE Transactions on Electromagnetic Compatibility, 49, 170-181, 2007.

Oprisan, D. and Rohling, H.: Analysis of Mutual Interference between Automotive Radar Systems, International Radar Symposium, Berlin, Germany, Session: Automotive Radar I, 2005.

Tullsson, B.: Topics in FMCW Radar Disturbance Suppression, Radar 97, Edinburgh, United Kingdom, 1-5, 1997.

Gresham, I., Jenkins, A., Egri, R., et al.: Ultra-Wideband Radar Sensors for Short-Range Vehicular Applications, IEEE Transactions on Microwave Theory and Techniques, 52, 2105-2122, 2004.

\section{Conclusion}

Mutual interference between automotive radar sensors is expected to become an increasingly important topic. This is due the fact that, on the one hand, automotive radar sensors 